\title{
The Crossing Angle in CLIC
}

\author{
D. Schulte, F. Zimmermann, CERN, Geneva, Switzerland
}

\begin{abstract}
Lower limits on the crossing angle at the interactionpoint (IP) of the compact linear collider (CLIC) are set by the multi-bunch kink instability, which, at $3 \mathrm{TeV}$ centre-ofmass energy, is strongly affected by coherent pairs. Aperture requirements for the spent beam introduce a second limitation. Finally, synchrotron radiation in the detector solenoid field, in the solenoid fringe field, and in the final quadrupole field imposes an upper limit on the crossing angle.
\end{abstract}

\section{INTRODUCTION}

At the CLIC centre-of-mass energy of $3 \mathrm{TeV}$ (for the parameters see table 1) the two beams must collide with a horizontal crossing angle $\theta_{c}$. Figure 1 sketches the area around the interaction point. The vertical scale is enhanced for better visibility. In the current base line final focus system [2] a distance $l^{\star}=2 \mathrm{~m}$ is foreseen between final quadrupole and interaction point (IP), but it may be possible to increase it. The length of the solenoid has not been specified yet; we assume $l_{\text {sol }}=6 \mathrm{~m}$.

First we will discuss the lower limits of the crossing angle which are given by the coherent pair creation and the multi-bunch kink instability, then the upper limits which are given by the effects of the main- and fringe-field of the solenoid.

\section{COHERENT PAIRS}

At the IP, due to the small spot size, each bunch produces very strong electro-magnetic fields that deform the oncoming bunch during the collision. The particles of this bunch emit hard photons, called beamstrahlung. In the strong field, these photons can turn into electron-positron pairs, the coherent pair creation. The particles produced are strongly deflected by the beam fields, so that they leave the interaction point at angles of the order of milli-radians. Since the total power in these pairs is a significant fraction

\begin{tabular}{lcc}
\hline variable & symbol & value \\
\hline beam energy & $E$ & $1.5 \mathrm{TeV}$ \\
particles per bunch & $N$ & $4 \times 10^{9}$ \\
hor./vert. rms emittance & $\gamma \epsilon_{x} / \gamma \epsilon_{y}$ & $0.68 / 0.02 \mu \mathrm{m}$ \\
hor./vert. rms spot size & $\sigma_{x 0}^{*} / \sigma_{y 0}^{*}$ & $43 / 1 \mathrm{~nm}$ \\
rms bunch length & $\sigma_{z}$ & $30 \mu \mathrm{m}$ \\
hor./vert. IP beta function & $\beta_{x}^{*} / \beta_{y}^{*}$ & $8 / 0.1 \mathrm{~mm}$ \\
distance between bunches & $\Delta s$ & $20 \mathrm{~cm}$ \\
\hline
\end{tabular}

Table 1: Basic interaction-point beam parameters for CLIC at $3 \mathrm{TeV}$ [1]. of the beam power, one has to avoid losing them in the region of the detector. This requires an exit hole for the spent beam with an opening angle of $10 \mathrm{mrad}$ around the nominal beam line [3]. Taking into account the outer radius of the last quadrupole in the incoming beam line of about $2 \mathrm{~cm}$ [4], one can conclude that the crossing angle needs to be at least $\theta_{c} \geq 20 \mathrm{mrad}$.

\section{MULTI-BUNCH KINK INSTABILITY}

Because of the small bunch spacing there will be parasitic crossings between outgoing and incoming beams inside the detector. If the beams collide with no angle or position error, and in the absence of a magnetic field, only horizontal kicks occur. Since they have the same direction for both beams, this only leads to a very small transverse shift of the IP for subsequent bunches, if the two beam intensities are the same.

For non-perfect beams, however, the parasitic collisions can lead to an instability [5]. Particularly severe is the case of two beams colliding with a vertical offset. Due to the beam-beam interaction, the first colliding bunches will leave the IP with a large vertical angle. This in turn leads to vertical offsets in the parasitic collisions which kick the incoming bunches such that their offset at the IP is increased. This effect can be strongly reduced by increasing the crossing angle $\theta_{c}$ between the two beams.

In the case of CLIC, the particles from coherent pair creation have also to be taken into account. If two beams collide with a vertical offset, the electrons of the coherent pairs

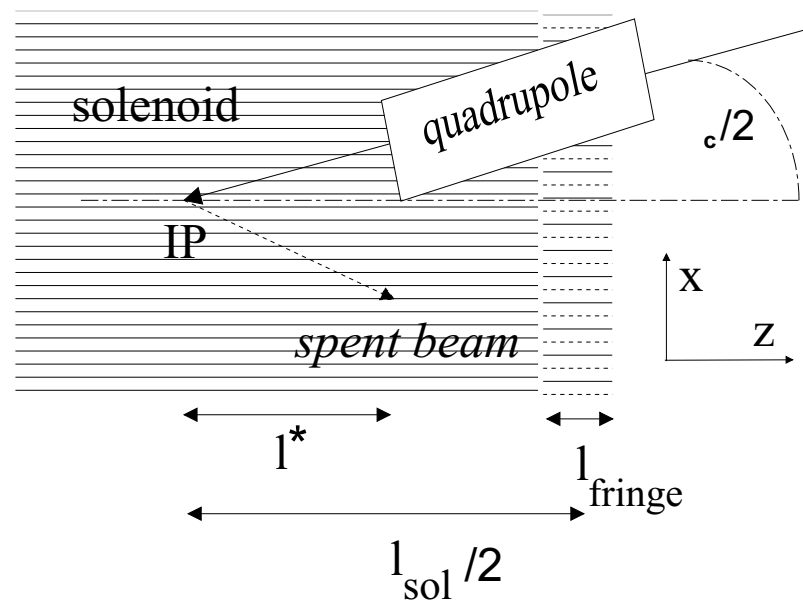

Figure 1: Sketch of the CLIC interaction region, showing the assumed superposition of the solenoid field, the solenoid fringe field and the final quadrupole magnet. 


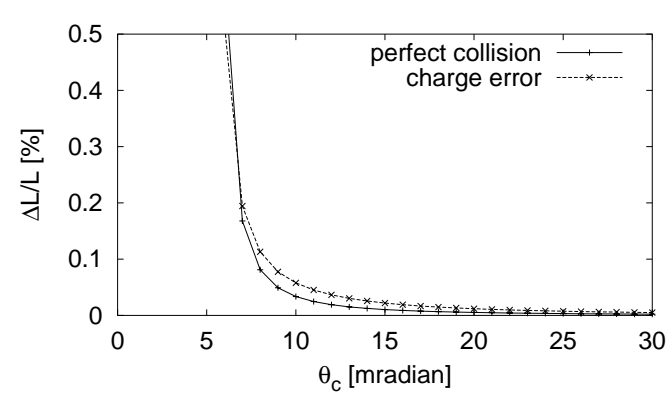

Figure 2: The luminosity loss as a function of the crossing angle $\theta_{c}$. In both cases the beams collide initially with no position or angle errors. In one case the bunch charge of one beam is only $90 \%$ of the nominal one.

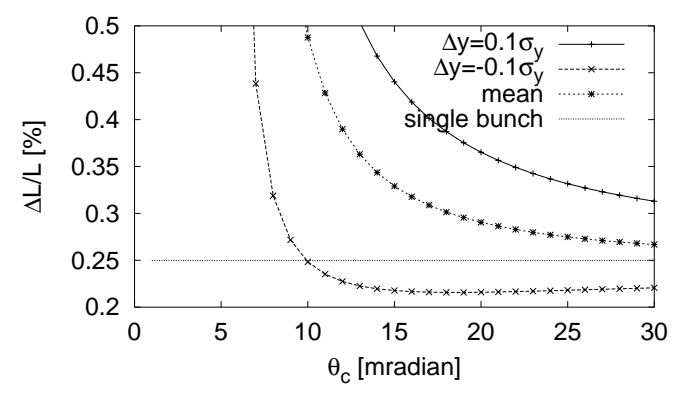

Figure 3: The luminosity loss as a function of the crossing angle $\theta_{c}$ for beams with an initial offset.

are deflected in one direction, the positrons in the other. The resulting dipole field kicks the incoming beam in the parasitic collisions with about the same strength and in the same direction as the field from the beam particles.

Of course, because of the solenoid field $B_{z}$ the particles do not travel on straight lines but rather on helices. In combination with the energy loss that the particles undergo at the IP and the production of coherent pairs, this leads to vertical deflections in the parasitic collisions. The vertical offset at the IP induced by this effect can be calculated as

$$
\Delta y \approx 0.3 \frac{\mathrm{GeV}}{\mathrm{Tm}} \frac{N_{f} r_{e}}{2 \gamma \theta_{c}} B_{z} \Delta s \frac{1}{E_{1}} \frac{n(n+1)}{2}
$$

Here, $N_{f}$ is the number of particles in the spent beam, $n$ is the number of parasitic crossings, $r_{e}$ is the classical electron radius, and $E_{1}$ is defined by the spectral density of particles in the spent beam $\rho(E)$ as

$$
\frac{1}{E_{1}}=\frac{1}{N_{f}} \int_{0}^{E_{0}} \rho(E)\left|\frac{1}{E}-\frac{1}{E_{0}}\right| d E
$$

Positrons in an electron bunch are treated by using a negative energy $E$ and vice versa. For CLIC, with $n=$ $l^{*} /(\Delta s / 2)=20$ we find $E \approx 280 \mathrm{GeV}$ and $\Delta y \approx$ $15 \mathrm{pm} \ll \sigma_{y}=1 \mathrm{~nm}$.

We have simulated the beam-beam collisions in the IP with Guinea-Pig [6] and written a tracking program for the parasitic collisions. Figure 2 shows the luminosity loss
$\Delta \mathcal{L} / \mathcal{L}$ as a function of the crossing angle $\theta_{c}$ for beams that collide initially without position or angle error. In one case the charge in one beam is only $90 \%$ of the nominal one. The losses are acceptable for $\theta_{c} \geq 10 \mathrm{mrad}$.

Figure 3 shows the luminosity loss as a function of the crossing angle for beams with an initial offset of $\Delta y=$ $\pm 0.1 \sigma_{y}$. We take the average of the two values and find that the multi-bunch effect increases the luminosity loss by a factor 2 with respect to the single bunch effect for $\theta_{c}=$ $10 \mathrm{mrad}$ and only by an acceptable $15 \%$ for $\theta_{c}=20 \mathrm{mrad}$.

\section{SOLENOID FIELD EFFECTS}

Due to the horizontal crossing angle, the longitudinal solenoid field gives rise to a vertical deflection and vertical dispersion at the interaction point. This dispersion and the additional energy spread induced by synchrotron radiation in the central solenoid field, the solenoid fringe field and in the final quadrupole, lead to an increase of the IP spot size.

For the effect of synchrotron radiation in the central solenoid field alone, a concise expression was derived by Irwin [7]:

$$
\frac{\Delta \sigma_{y}^{2}}{\sigma_{y 0}^{2}}=\frac{1}{20} \frac{c_{u} r_{e} \lambda_{e}}{\sigma_{y 0}^{2}}\left(\frac{B_{z} \theta_{c} l^{*} \gamma}{2(B \rho)}\right)^{5}
$$

where $c_{u}=55 /(24 \sqrt{3}),(B \rho)$ is the magnetic rigidity, and $\sigma_{y 0}^{*}=1 \mathrm{~nm}$ the ideal spot size without synchrotron radiation.

The beam size increase due to synchrotron radiation in the final quadrupole and energy-dependent focusing is called the Oide effect $[8,9]$. The beam size increase due to this effect, taking into account also the horizontal motion inside the quadrupole, can be expressed as [7]:

$$
\begin{aligned}
& \Delta \sigma_{y}^{2} \approx \frac{15 \sqrt{\pi} c_{u} r_{e} \lambda_{e} \gamma^{5} \sigma_{y}^{\prime * 2}}{32} \int d s L_{y}^{c}(s)^{2}\left|\frac{1}{\rho}\right|^{3} \times \\
& {\left[\sigma_{x}^{\prime * 2} R_{12}(s)^{2}+\sigma_{y}^{* * 2} R_{34}(s)^{2}\right]^{1 / 2} \times} \\
& {\left[\sigma_{x}^{*{ }^{2}} R_{12}(s)^{2}+7 \sigma_{y}^{\prime * 2} R_{34}(s)^{2}\right]}
\end{aligned}
$$

where $\rho$ is the local bending radius, $R_{12}$ and $R_{34}$ the rays originating from the IP, and $L_{y}^{c}$ the chromatic length given as

$$
L_{y}^{c}=\int d s \kappa(s) R_{34}(s)^{2}=\int d s \kappa(s) \beta_{y}(s) .
$$

where $\kappa$ is the strength of the quadrupole in units of $\mathrm{m}^{-2}$. For the baseline final focus with a quadrupole strength $\kappa=$ $0.089 \mathrm{~m}^{-2}$, length $l_{q}=4.8 \mathrm{~m}$, and $l^{*}=2 \mathrm{~m}$, we evaluated Eq. (4) numerically and found $\sigma_{y}^{*} / \sigma_{y 0}^{*} \approx 1.13$.

The local bending radius in the solenoid fringe field is taken to be $\rho^{-1}=\left(\rho_{x}^{-2}+\rho_{y}^{-2}\right)^{1 / 2}$ where

$$
\begin{gathered}
\frac{1}{\rho_{y}}=\frac{B_{z}}{2(B \rho)} \frac{1}{l_{\text {fringe }}}\left(x_{\text {off }}+x\right), \\
\frac{1}{\rho_{x}}=-\frac{B_{z}}{2(B \rho)} \frac{1}{l_{\text {fringe }}} y,
\end{gathered}
$$


$x$ and $y$ are the particle coordinates with respect to the design trajectory, $l_{\text {fringe }}$ denotes the length of the fringe-field region, and $x_{\text {off }}$ is the beam offset at the fringe from the centre line of the solenoid. It might seem attractive to align the solenoid axis with the position of the beam at the fringe so that $x_{\text {off }}=0$. However, in that case the vertical dispersion generated by the fringe no longer cancels the vertical dispersion generated by the inner solenoid field. Below, we assume the conventional offset $x_{\text {off }}=l^{*} \sin \theta$.

We have written a simple tracking program to study the combined effect of solenoid field, solenoid fringe field and final quadrupole magnet. These three fields may be partially superimposed. We consider the case that the solenoid extends beyond the quadrupole end face, as illustrated in Fig. 1. The photon emission probability and the energy of synchrotron photons are calculated by a Monte Carlo program as described in Ref. [10]. Inside the central solenoid field, the vertical coordinate with respect to an on-energy reference particle varies as $d^{2} y / d s^{2}=\delta /(1+$ $\delta) B_{z} \sin \theta /(B \rho)$, where $\delta$ denotes the relative momentum deviation. Synchrotron radiation is computed using the local inverse bending radius $1 / \rho=-\left(B_{z} \sin \theta /(B \rho)\right)$.

Figure 4 shows the simulated effect of the inner solenoid field only. The prediction of Eq. (3) is also shown.

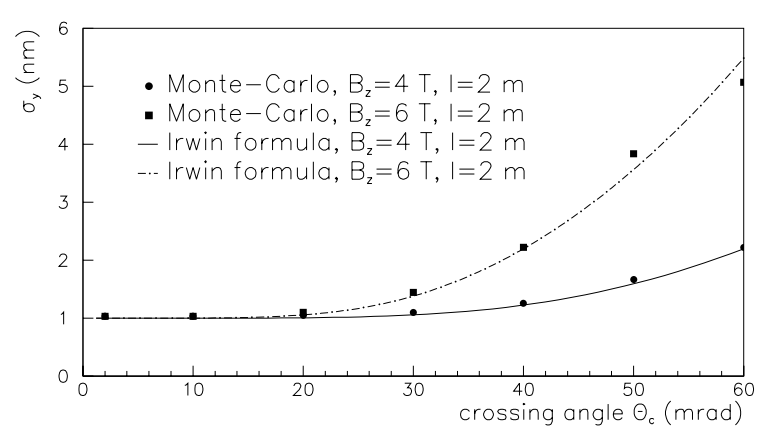

Figure 4: Vertical spot size increase $\Delta \sigma_{y}^{*} / \sigma_{y}^{*}$ due to synchrotron radiation and vertical dispersion in the solenoid field as a function of the crossing angle. Compared are the results of a Monte-Carlo tracking simulation and an analytical estimate [7], for two different values of the solenoid field.

Figure 5 illustrates the combined effect of solenoid, solenoid fringe and final quadrupole fields. Two curves, corresponding to $l_{\text {fringe }}=0.2 \mathrm{~m}$ and $l_{\text {fringe }}=1 \mathrm{~m}$ are shown for a 4-T and a 6-T solenoid field. Figure 6 illustrates the importance of the solenoid fringe field.

In all of the cases considered, the average number of photons emitted per electron is about 1.5.

\section{CONCLUSION}

To avoid secondary background due to the coherent pairs created at the IP, a minimum crossing angle $\theta_{c} \geq$ $20 \mathrm{mrad}$ is required for CLIC. This crossing angle also leads to acceptable multi-bunch effects due to parasitic collisions. Synchrotron radiation in the final quadrupole

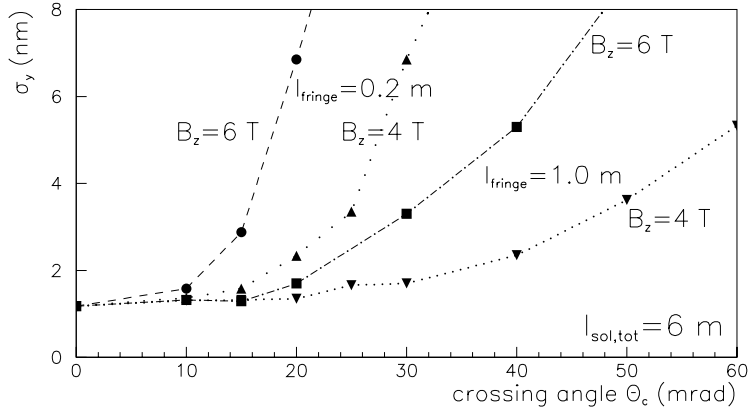

Figure 5: Vertical spot size $\sigma_{y}^{*}$ as a function of the crossing angle, revealing the effect of synchrotron radiation in the final quadrupole magnet, solenoid fringe, and central solenoid fields. Results are shown from a tracking simulation for $4 \mathrm{~T}$ and $6 \mathrm{~T}$ solenoid field with two different lengths of the fringe field.

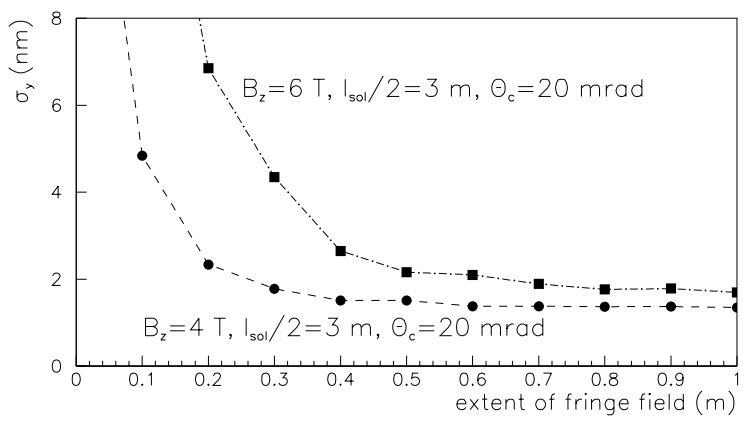

Figure 6: Vertical spot size $\sigma_{y}^{*}$ as a function of fringe field extent, including the effect of synchrotron radiation in the final quadrupole magnet, solenoid fringe, and central solenoid fields. Results are shown from a tracking simulation, for $\theta_{c}=20 \mathrm{mrad}$ and solenoid fields of 4 and $6 \mathrm{~T}$.

and the solenoid increases the vertical spot size at the IP. We have calculated that this growth is acceptable for $\theta_{c} \leq 20 \mathrm{mrad}$ in a $4 \mathrm{~T}$ solenoid field if the local fringe field is sufficiently small.

\section{REFERENCES}

[1] G. Guignard (ed.) et al., CERN-2000-008 (2000).

[2] F. Zimmermann, et al., Proc. EPAC 2000 Vienna, and CERNSL-2000-057 AP.

[3] D. Schulte. CERN/PS 99-017 (LP) (1999).

[4] M. Aleksa and S. Russenschuck. Private communication.

[5] P. Chen and K. Yokoya. KEK Preprint 91-2 (1991).

[6] D. Schulte. ICAP98, Monterey, CA., USA, September 1998.

[7] J. Irwin, in the Zeroth Order Design Report of the Next Linear Collider, Chapter 11, SLAC-R-0474 (1996).

[8] K. Oide, Phys. Rev. Lett. 61, 1713 (1988).

[9] K. Hirata, K. Oide and B. Zotter, Phys. Lett. B224, 437 (1989).

[10] G. Roy. Nucl. Instr. Methods A298 p. 128 (1990). 